MATEC Web of Conferences 12, 10002 (2014)

DOI: $10.1051 /$ matecconf $/ 20141210002$

(C) Owned by the authors, published by EDP Sciences, 2014

\title{
VHCF of spray formed hypereutectic aluminium silicon alloy
}

\author{
M. Fitzka ${ }^{1, a}$, H. Mayer ${ }^{1}$, R. Schuller ${ }^{1}$, S. Stanzl-Tschegg ${ }^{1}$, T. Przeorski ${ }^{2}$ and P. Krug $^{3}$ \\ ${ }^{1}$ Institute of Physics and Materials Science, BOKU, Austria \\ ${ }^{2}$ PEAK Werkstoff GmbH, Germany \\ ${ }^{3}$ Institute of Automotive Engineering, Cologne University of Applied Sciences, Germany
}

\section{Materials and method}

Ultrasonic fatigue experiments in the very high cycle fatigue (VHCF) regime are performed with spray-formed hypereutectic aluminium silicon alloy DISPAL ${ }^{\circledR}$ S232-T6x. DISPAL exhibits excellent mechanical properties with low weight and can achieve higher stiffness, greater high temperature strength, excellent wear resistance and a reduced coefficient of thermal expansion compared to cast or wrought aluminium alloys. T6x indicates peak ageing with improved ductility.

In the present investigation specimens are cycled in resonance in constant (CA) and variable amplitude (VA) tests at ultrasonic frequency in the high cycle fatigue (HCF) and VHCF regime up to $2 \times 10^{10}$ cycles [1]. The cycling frequency of approximately $20 \mathrm{kHz}$ allows accumulating fatigue data within reasonable testing times. Means of non-linear acoustics are employed for detecting early stages of fatigue damage and for monitoring vibration properties during fatigue life. Miner calculation and lifetime prediction is performed to describe mean VA lifetimes. Early failures caused by large materials defects are considered by using an adapted crack growth model.

\section{Results and discussion}

Figure 1 (left panel) shows the fatigue properties in CA experiments for stress amplitudes $\Delta \sigma / 2$ between $296 \mathrm{MPa}$ and $139 \mathrm{MPa}$, yielding lifetimes between $3.2 \times 10^{5}$ and $7.6 \times 10^{9}$ cycles. Lifetimes in VA tests for maximum stress amplitudes $\Delta \sigma_{\max } / 2$ between $296 \mathrm{MPa}$ and $185 \mathrm{MPa}$ were found between $4.3 \times 10^{7}$ and $1.7 \times 10^{10}$. Additionally, means of non-linear acoustics are employed in computer control and data acquisition for monitoring and analysis of the specimens' vibration properties in CA experiments [2]. The method allows deriving the resonance frequency as well as the non-linearity parameter $\beta_{\text {rel }}$, which may serve for early detection of fatigue damage [3]. Changes in resonance frequency and non-linearity parameter $\beta_{\text {rel }}$ are indicators for microstructural changes and are used to monitor progress of fatigue damage in the VHCF regime. Figure 1 (right panel) shows an exemplary course of resonance frequency and $\beta_{\text {rel }}$ versus number of cycles of a specimen that failed after $1.3 \times 10^{9}$ cycles at $\Delta \sigma / 2=162 \mathrm{MPa}$. From the very beginning of the test, the resonance frequency slowly decreases, while $\beta_{\text {rel }}$ continuously increases. The appearance of increasing numbers and lengths of fatigue cracks affects resonance frequency as a consequence of increased specimen compliance. This indicates that fatigue cracks are

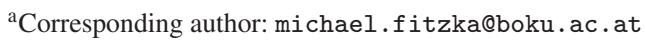

This is an Open Access article distributed under the terms of the Creative Commons Attribution License 4.0, which permits unrestricted use, distribution, and reproduction in any medium, provided the original work is properly cited. 

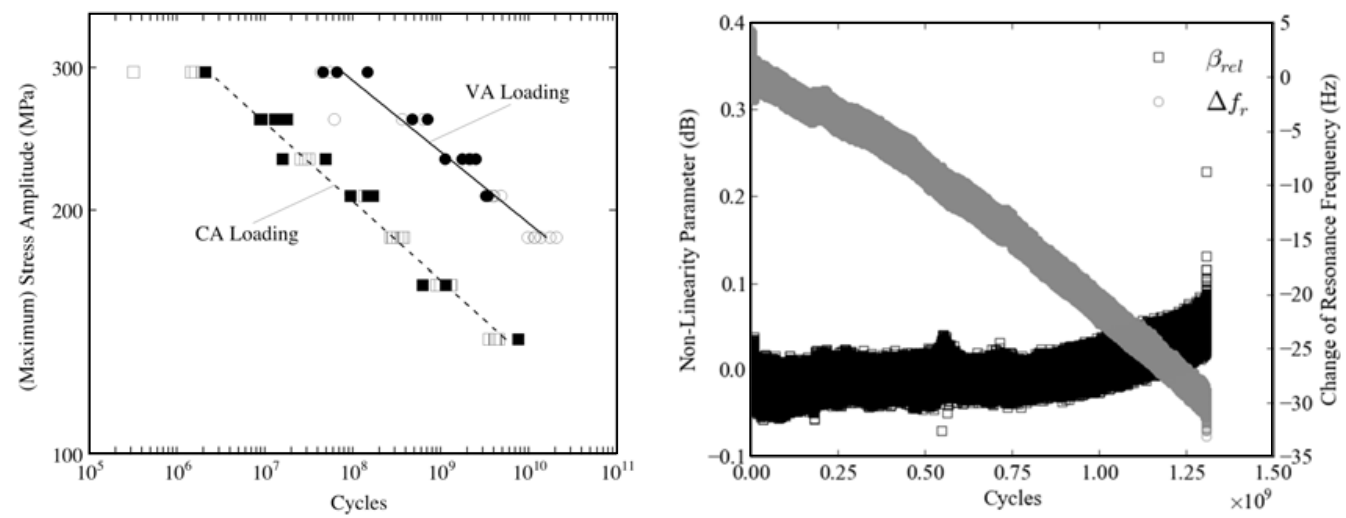

Figure 1. Fatigue lifetimes for CA (squares) and VA loading (circles) (left), open symbols indicate crack initiation in the interior of the specimen, solid symbols indicate surface crack initiation; (right) variation of resonance frequency $\left(\Delta \mathrm{f}_{\mathrm{r}}\right.$, grey) and non-linearity parameter $\left(\beta_{\text {rel }}\right.$; black) versus numbers of cycles in a CA experiment with $\Delta \sigma / 2=162 \mathrm{MPa}$ and $1.3 \times 10^{9}$ cycles to failure.

formed virtually from the first load cycle and that fatigue lifetime of DISPAL ${ }^{2} 32-T 6 x$ is the number of cycles necessary to propagate cracks to rupture, even in the VHCF regime.

Miner calculation delivers damage sums between 0.5 and 0.9 and may be used to predict mean lifetimes. Failures in the material are caused by voids, oxides and distributed inhomogeneities. Large voids and oxides are the main reasons for early failures, which cannot be covered with the Miner calculation. Lifetime prediction can be performed using an adapted Paris-law for the propagation of (small) cracks and assuming crack initiating defects as initial cracks. Besides mean lifetimes, early failures can be successfully predicted. Moreover, the crack propagation model is a physically valid description of the fatigue process in DISPAL ${ }^{\circledR}$ S232-T6x as shown with the non-linear acoustics analysis.

\section{Conclusions and outlook}

It has been shown that for the spray-formed hypereutectic aluminium silicon alloy DISPAL® S232T6x second order harmonics and resonance frequency may be linked to progressing fatigue damage. Monitoring resonance frequency shows an increase of specimen compliance from the beginning of fatigue cycling. It is concluded that fatigue lifetime of DISPAL® 232-T6x, even in the VHCF regime, is mainly determined by the number of cycles necessary to propagate cracks to rupture. This can be used in a physically sound model to describe fatigue lifetime.

The most recent developments concern increasing the accuracy and resolution of this monitoring method. First results show, that resolution of resonance frequency far better than $1 \mathrm{~Hz}$ allows detecting early material changes even if high strength materials are stressed in the VHCF regime. Crack initiating in secondary phase particles in aluminium alloy 2024-T351, for example, affect resonance frequency if it is monitored with sufficient resolution.

\section{References}

[1] M. Fitzka, H. Mayer, R. Schuller, S.E. Stanzl-Tschegg, T. Przeorski, P. Krug, Variable amplitude loading of spray-formed hypereutectic aluminium silicon alloy DISPAL $®$ S232 in the VHCF regime, Fatigue Fract. Eng. M., in print (2014) 
[2] H. Mayer, M. Fitzka, R. Schuller, Constant and variable amplitude ultrasonic fatigue of 2024-T351 aluminium alloy at different load ratios, Ultrasonics 53 (2013) 1425-1432

[3] A. Kumar, C.J. Torbet, J.W. Jones, T.M. Pollock, Nonlinear ultrasonics for in situ damage detection during high frequency fatigue, Journal of Applied Physics 106 (2009) 024904 